
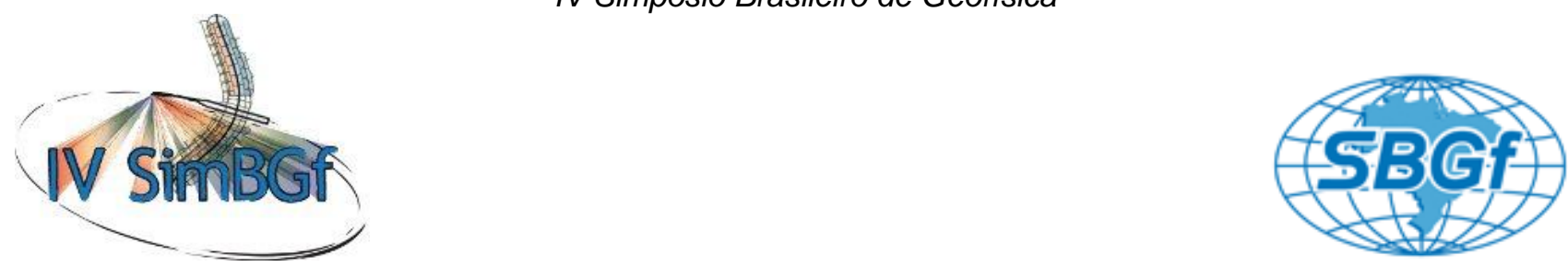

\title{
PreSTM E CRS-2D DE DADOS REAIS DA BACIA DO AMAZONAS
}

Danilo Galvão Ferreira, CPGeo e UFPA (Brasil)

Rafael dos Santos Furtado, CPGeo e UFPA (Brasil)

German Garabito Callapino, UFRN (Brasil)

Copyright 2010, SBGf - Sociedade Brasileira de Geofísica

Este texto foi preparado para a apresentação no IV Simpósio Brasileiro de Geofísica Brasília, 14 a 17 de novembro de 2010. Seu conteúdo foi revisado pelo Comité Técnico do IV SimBGf, mas não necessariamente representa a opinião da SBGf ou de seus associados. É proibida a reprodução total ou parcial deste material para propósitos comerciais sem prévia autorização da SBGf.

\section{Abstract}

Among the several problems in prospecting for oil and gas in the Amazon region, diabase sills occurring in the Amazonas Basin (Brazil), in spite of its role in the maturation of the organic matter that originated all hydrocarbons accumulations known so far, still poses difficulties for exploration seismic, causing anomalous seismic wave spreading, multiple reflactions and pull-up and pull-down effects, consequently rising the exploration risk. In this context, the present work then presents the results of modern techniques (PreSTM) and of nonconventional (CRS) seismic processing of the survey lines 0204-RL-0053, 0204-RL-0057 and 0204-RL-0058 acquired in the Amazonas Basin, with the objective of enhancing the seismic images of these cited sections.

\section{Introdução}

A Bacia intracratônica do Amazonas como um todo, Alto Amazonas (Solimões), Médio e Baixo Amazonas (Amazonas), está inserida no contexto da floresta Amazônica e implantada sobre o Cráton Amazônico de idade e evolução pré-Ciclo Brasiliano. Localizada no interior da Plataforma Sul-Americana, a "Bacia do Amazonas", atualmente, corresponde às antigas bacias do Médio e Baixo Amazonas, enquanto que a denominação "Bacia do Solimões" corresponde à Bacia do Alto Amazonas (CAPUTO 1984). Em estudos mais antigos, o nome "Bacia do Solimões" se aplicava à cobertura cenozóica do oeste da Amazônia, mas Caputo (1984) sugeriu a substituição do nome Alto Amazonas por Solimões, devido a esta apresentar uma história tectono-sedimentar diferenciada da Bacia do Amazonas.

Eventos tectono-termais, caracterizados por pulsos magmáticos que atingiram praticamente todas as bacias paleozóicas brasileiras, foram os responsáveis pela injeção de material magmático no interior da bacia do Amazonas dando origem as soleiras de diabásio. Apesar dessas soleiras que se encontram intrudidas nas rochas paleozóicas da bacia do Amazonas terem exercido um papel importante no processo de maturação da matéria orgânica, sob o ponto de vista exploratório, a ocorrência de rochas magmáticas nas porções internas de bacias sedimentares são uma problemática para a exploração geofísica por se tratarem de rochas que dificultam a penetração das ondas sísmicas (EIRAS e WANDERLEY FILHO, 2002). Com o propósito de melhor entender e suprimir os efeitos negativos na qualidade das imagens sísmicas da Bacia do Amazonas, o presente trabalho tem como objetivo principal produzir imagens sísmicas de melhor qualidade por meio do reprocessamento de linhas sísmicas utilizando técnicas modernas de tratamento de dados sísmicos empregadas na atualidade pela indústria como a migração pré-empilhamento (PreSTM) e técnicas especiais como o método não convencional de empilhamento por superfície de reflexão comum (CRS).

O método de migração escolhido para integrar o presente trabalho foi a migração Kirchhoff. Esse método baseia-se na soma ou espalhamento ponderado de amplitudes ao longo de curvas de tempo de trânsito de difração ou curvas isócronas. A migração Kirchhoff foi utilizada tanto nas seções migradas com a técnica pré-empilhamento, quanto nas seções migradas após empilhamneto CRS.

\section{Metodologia/ Problema Investigado}

As linhas sísmicas 0204-RL-0053, 0204-RL-0057 e 0204RL-0058, mostradas na Figura 1, fazem parte de um conjunto de dados adquiridos da Agência Nacional do Petróleo, Gás Natural e Biocombustíveis (ANP) pelo Instituto de Geociências da Universidade Federal do Pará (IG-UFPA) para fins de desenvolvimento acadêmico.

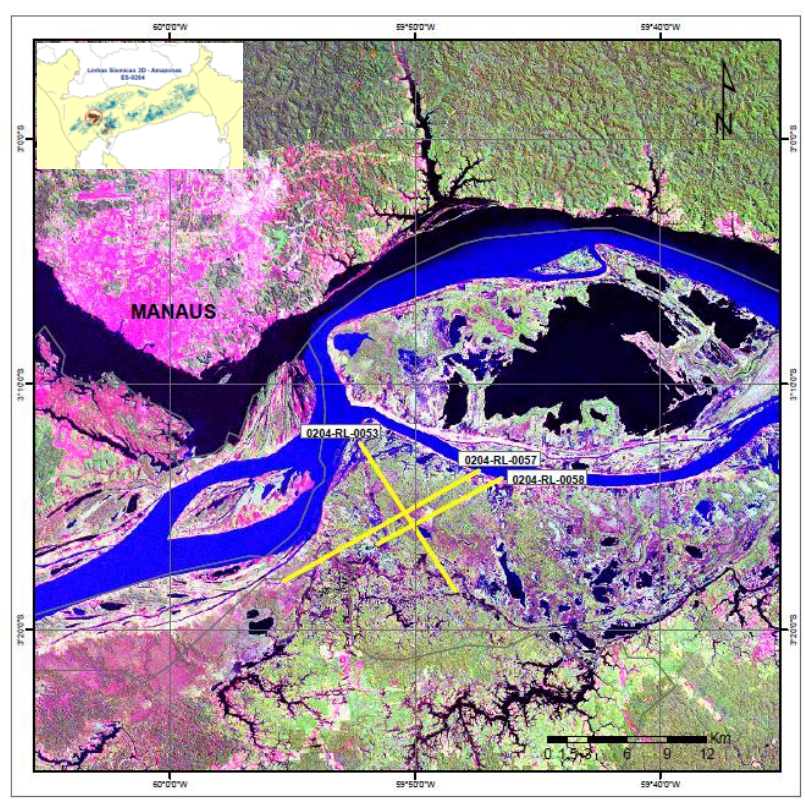

Figura 1 - Mapa de localização das linhas sísmicas 0204-RL0053, 0204-RL-0057 e 0204-RL-0058 destacadas em amarelo. 
Toda a etapa de pré-processamento dos dados sísmicos, análise de velocidades e migração pré-empilhamento em tempo foi realizada com o aplicativo comercial ProMAX®, versão 2003.19.1.1, da Landmark Graphics Corporation, empresa da Halliburton Ltda. Já na etapa de aplicação do método CRS utilizou-se o algoritmo desenvolvido por Garabito et al. (2001) e Garabito (2001). Também foi utilizado o programa ArcGIS, versão 9.2, para a localização e o georreferenciamento das três linhas usadas neste trabalho.

Como já foi citado anteriormente as soleiras de diabásio presentes na Bacia do Amazonas e em todas as bacias paleozóicas brasileiras, foram geradas pelo Evento Penatecaua no Jurotriássico durante a abertura do Atlântico Norte (ISSLER et al., 1974) e atuaram como fonte extra de calor para o processo de transformação da matéria orgânica em óleo e gás, assim como no craqueamento de óleo na bacia. Por outro lado, essas soleiras de diabásio são um problema para geofísica de exploração. A problemática está na dificuldade em processar dados sísmicos de baixa qualidade proveniente de área com feições geológicas complexas associadas à ocorrência de rochas magmáticas, como as da bacia do Amazonas. Esse obstáculo surge do fato de que as soleiras de diabásio podem deteriorar a qualidade das seções sísmicas, por causarem a perda do sinal, a geração de múltiplas e o espalhamento do sinal, prejudicando assim, a interpretação dos dados pela formação de falsas estruturas (EIRAS; WANDERLEY FILHO, 2002).

\section{PreSTM}

A migração pré-empilhamento é a designação dada ao método de migração aplicada em seções pré-empilhadas quando a mesma está baseada na configuração de afastamento comum, ou seja, a migração préempilhamento pode ser considerada como um desenvolvimento da técnica pós-empilhamento considerando o afastamento não-nulo. Para tal, cada traço sísmico de uma seção Common offset é individualizado pelo valor da coordenada de ponto médio $\left(x_{m}\right)$.

Logo, considerando está configuração, matematicamente o processo de migração Kirchhoff é representado pela seguinte integral:

$$
V(M)=\int W\left(x_{m}, M\right) D^{2} \gamma_{z}\left[U\left(x_{m}, t=\tau_{b}\left(x_{m}, M\right)\right)\right] d x_{m}
$$

sendo $W$ a função peso ponderada as amplitudes de migração, $x_{m}$ o ponto médio, $M \circ$ ponto imagem, $D$ a derivada de meio tempo aplicado aos dados de entrada para restaurar a forma da wavelet.

Neste trabalho, a migração Kirchhoff pré-empilhamento considera meios homogêneos e com velocidade constante, em que os tempos de difrações são calculados por:

$$
\tau_{p}\left(x_{m}, M\right)=\frac{1}{v} \sqrt{\left(x_{0}-x_{m}+h\right)^{2}+z^{2}}+\sqrt{\left(x_{0}-x_{m}-h\right)^{2}+x^{2}}
$$

sendo $x_{0}$ a projeção horizontal das coordenadas do ponto imagem $M$.

Deste modo, a curva de tempos de difração para o ponto $M$ em uma seção Common offset é traçada variando-se as coordenadas $x_{m}$ dos pontos médios. Sobre esta curva são reunidas as amostras, que após serem multiplicadas pelo peso $W$ são somadas e o resultado atribuído ao ponto $M$ da seção de saída.

Supondo-se que se tenha uma amostragem contínua tanto em $h$ quanto em $x_{m}$, pode-se calcular o tempo de difração para um ponto $M$ em subsuperfície gerando assim a pirâmide de tempos de difração (Figura 2) ou pirâmide de Quéops (CLAERBOUT, 1985).

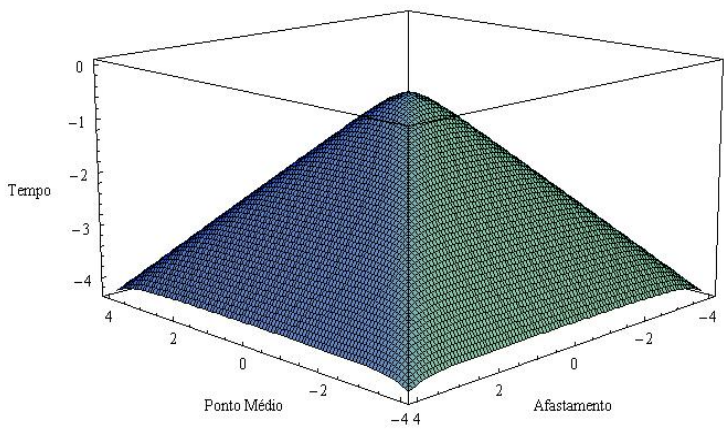

Figura 2 - Pirâmide de tempos de difração formada pelas trajetórias de empilhamento para uma seção Common offset. Fonte: Adaptado de Biondi (2006).

\section{CRS}

O método de empilhamento por superfície de reflexão comum (CRS - common reflection surface) é uma técnica de imageamento, que faz parte do grupo de métodos alternativos de empilhamento de dados sísmicos que não dependem do conhecimento a priori do modelo de velocidades do meio. O método CRS simula uma seção de afastamento nulo (ZO - zero offset), utilizando dados de cobertura múltipla, através da soma dos eventos sísmicos ao longo das superfícies de empilhamento associadas a cada ponto de amostragem da seção ZO. Estas seções ZO simuladas apresentam resolução lateral e relação sinal/ruído melhores que as apresentadas pelos métodos convencionais. Isso ocorre porque o método CRS admite reflexões associadas com um elemento refletor em subsuperfície, que é caracterizado pela posição, inclinação e curvatura local do refletor, diferente da técnica de empilhamento por ponto médio comum (CMP- Common Midpoint), que considera reflexões correspondentes a um único ponto refletor em profundidade.

\section{Operador CRS}

O empilhamento convencional de dados sísmicos em configuração CMP é realizado com aproximações hiperbólicas do tempo de trânsito. No empilhamento CRS 
não é diferente, de modo que este método utiliza aproximações do tempo de trânsito tri-paramétricas de segunda ou mais ordem. No presente trabalho, o empilhamento CRS utilizou a aproximação hiperbólica de segunda ordem através do trio de atributos cinemáticos $\left(\beta_{0}, R_{N}\right.$ e $\left.R_{N I P}\right)$ das ondas NIP e $N$, também denominados parâmetros de empilhamento CRS (TYGEL et al., 1997).

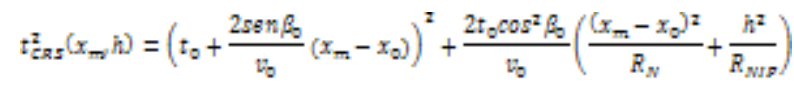

sendo $t_{0}$ o tempo duplo do raio central de afastamento nulo e $\mathbb{v}_{0}$ a velocidade próxima a superfície entorno do ponto de emergência do raio central. As coordenadas $x_{m}=\left(x_{G}+x_{s}\right) / 2$ e $h=\left(x_{G}-x_{5}\right) / 2$ são os pontos médio e o meio-afastamento entre a fonte e o receptor, respectivamente, sendo $x_{5}$ e $x_{G}$ as coordenadas horizontais do par fonte receptor. A equação (3) determina uma curva ou uma superfície de empilhamento CRS, que é independente do macro-modelo de velocidades. Na Figura 3 é ilustrada a superfície de empilhamento definida pela aproximação do tempo de trânsito CRS para o modelo composto por duas camadas homogêneas sobre um semi-espaço. Na parte superior, as curvas de tempo de trânsito das reflexões primárias referentes à segunda interface são representadas pelas linhas de cor azul, sob o domínio $\left(x_{m}, h\right)$. Ainda na parte superior a superfície CRS é representada pelas linhas de cor vermelha definidas sobre o ponto de amostragem $P_{0}$, o qual é associado à reflexão primária no ponto $R$ sobre 0 segundo refletor.

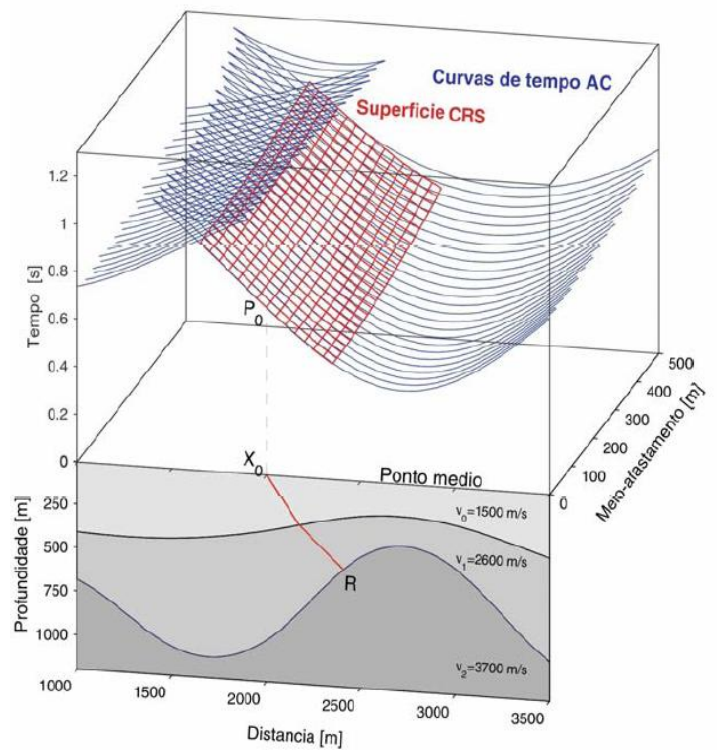

Figura 3 - Modelo composto por duas camadas homogêneas sobre um semi-espaço. Na parte inferior, encontra-se o raio de incidência normal (linha vermelha), ou raio de afastamento nulo. $\mathrm{Na}$ parte superior, as linhas de cor azul correspondem à superfície de cobertura múltipla, e as linhas de cor vermelha definem a superfície CRS, ambas associadas às reflexões da segunda interface. Fonte: (Garabito, 2001).
O processamento sísmico CRS dos dados reais da Bacia do Amazonas foi realizado através da estratégia de busca global-local (GARABITO, 2001; PASCHOAL JR., 2005), conforme processos descritos no fluxograma da Figura 4. Após alguns testes, foram escolhidos de forma empírica os parâmetros de abertura do operador, definidos de forma a fornecer uma melhor continuidade lateral dos eventos. Depois de determinado os parâmetros de processamento, o empilhamento CRS é realizado de modo automático. Vale ressaltar que no presente trabalho só serão mostradas as seções afastamento nulo migradas após empilhamento CRS, uma vez que, cada etapa do processamento gera resultados que são usados nas etapas posteriores, quer dizer, os resultados da primeira etapa são utilizados na segunda etapa e os resultados dessas duas etapas são usados na terceira e última etapa, que produz os resultados finais apresentados neste trabalho.

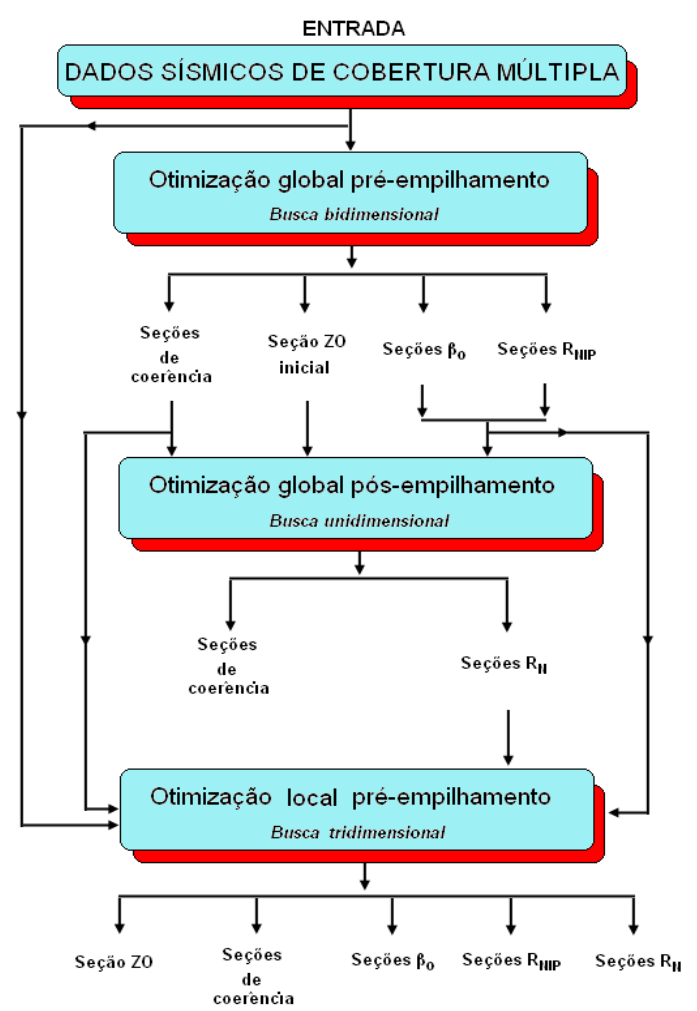

Figura 4 - Fluxograma simplificado das etapas do processamento sísmico CRS através da estratégia de busca global-local. Fonte: Modificado de Garabito (2001).

\section{Resultados}

As seções migradas finais com as duas técnicas de processamento sísmico (PreSTM e CRS) foram organizadas visando uma melhor comparação entre os dois processos, de modo que as Figuras 5 e 6 representam as seções PreSTM e CRS da linha 02040053, respectivamente. As Figuras 7 e 8 representam as seções PreSTM e CRS da linha 0204-0057, respectivamente e, finalmente, as Figuras 9 e 10 são referentes as seções PreSTM e CRS da linha 0204-0058, respectivamente. 
As duas seções sísmicas, PreSTM e CRS, correspondentes à linha 0204-RL-0053, Figuras 5 e 6, respectivamente, apresentam boa resolução e continuidade lateral dos refletores, principalmente no intervalo de 0.4 a $1 \mathrm{~s}$. Não obstante, ponderou-se que a seção migrada após o empilhamento CRS alcançou maior continuidade lateral dos eventos de reflexões profundos, assim como melhor definição dos refletores localizados nas partes rasas. Esta melhora na seção da referida linha sísmica pode ser observada, principalmente, entre os CDPs 230 a 250 e 540 a 550 e no intervalo de 0.4 a $1.1 \mathrm{~s}$, onde os refletores apresentam-se com melhor resolução na seção processada com o método CRS.

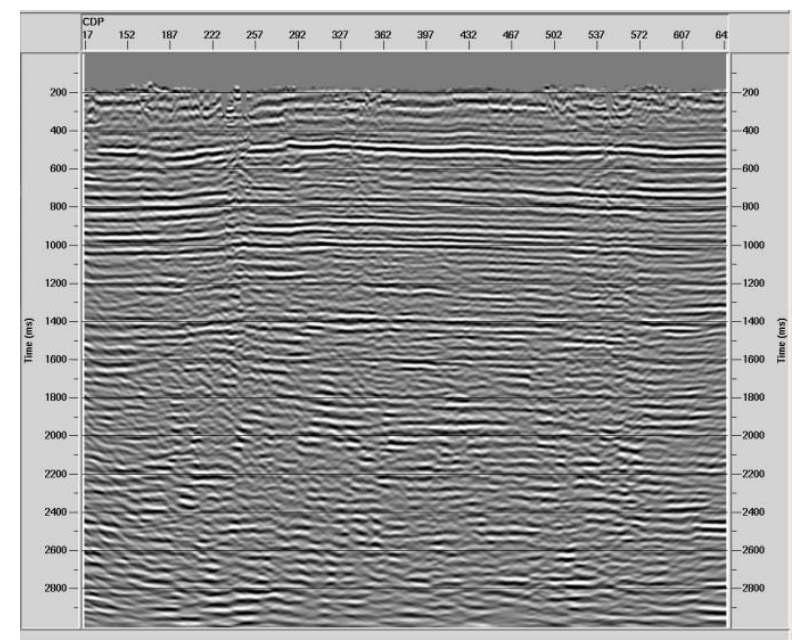

Figura 5 - Seção migrada com a técnica pré-empilhamento em tempo da linha 0204-RL-0053

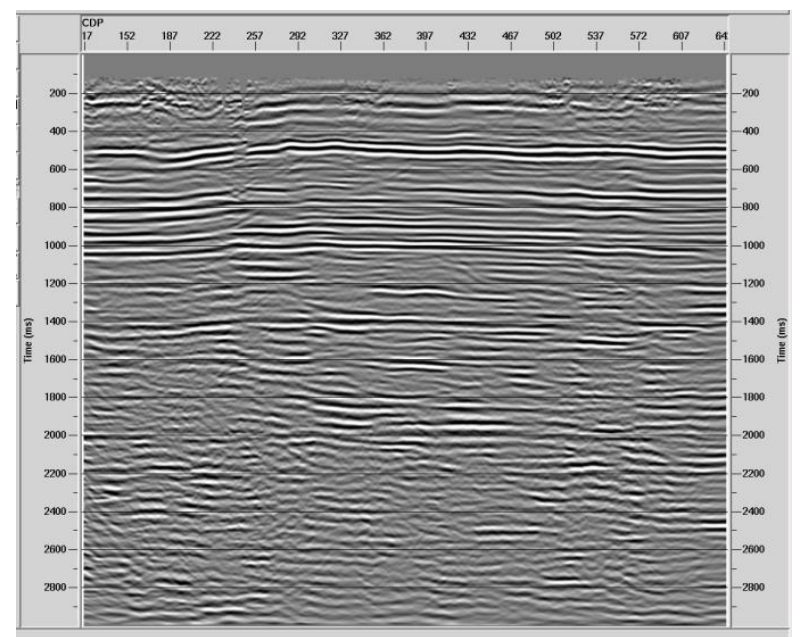

Figura 6 - Seção migrada em tempo após empilhamento CRS da linha 0204-RL-0053.

A grande quantidade de "tiros" perdidos na fase de aquisição (faixa sem reflexão entre os CDPs 320 e 360) e a baixa qualidade dos dados sísmicos da linha 0204-RL0057, devido à baixa razão sinal-ruído dos traços e a baixa cobertura, comprometeram tanto a seção PreSTM como a seção migrada após empilhamento CRS, Figuras 7 e 8, respectivamente. Na seção migrada com a técnica pré-empilhamento observa-se descontinuidades laterais dos eventos de reflexão em várias partes da seção e má resolução dos refletores abaixo de $0.8 \mathrm{~s}$. Apesar dos problemas da baixa razão sinal-ruído dos traços e a baixa cobertura que comprometem os processos automáticos de coerência, a seção migrada após o empilhamento CRS apresentou uma significativa melhora na definição e continuidade lateral dos eventos de reflexão profundos, abaixo de $0.8 \mathrm{~s}$.

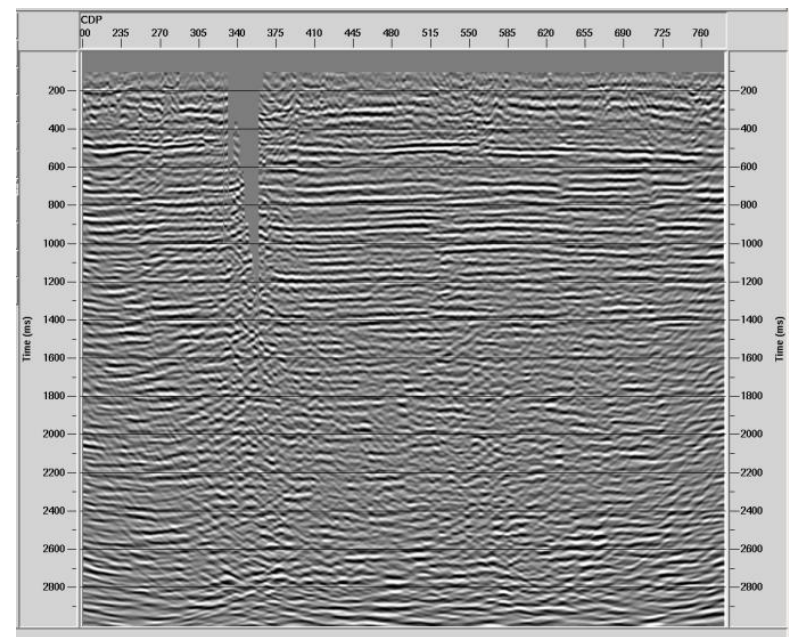

Figura 7 - Seção migrada com a técnica pré-empilhamento em tempo da linha 0204-RL-0057.

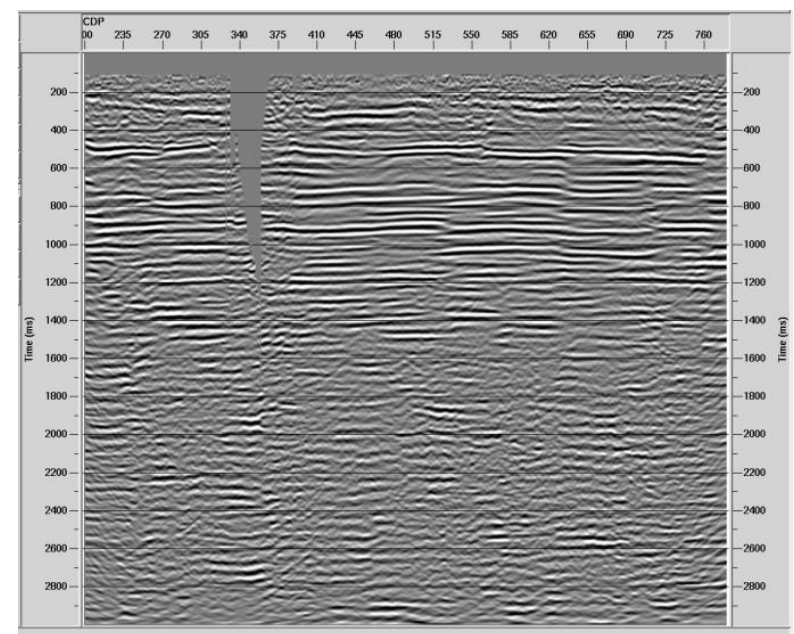

Figura 8 - Seção migrada em tempo após empilhamento CRS da linha 0204-RL-0057.

Assim como nas seções da linha 0204-RL-0053, ambas as seções correspondentes à linha 0204-RL-0058, seção PreSTM e CRS, Figuras 9 e 10, respectivamente, apresentaram continuidade lateral dos eventos de reflexão e boa resolução. Mas, observou-se que a seção migrada após a técnica de empilhamento CRS obteve melhor definição e maior continuidade lateral dos eventos de reflexão mais profundos, principalmente no intervalo de 0.6 a $1 \mathrm{~s}$. 


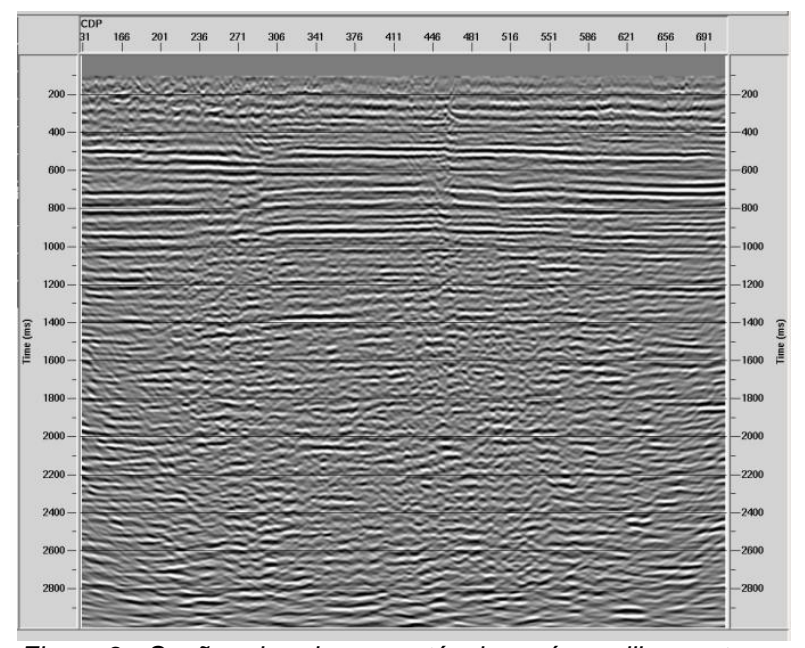

Figura 9 - Seção migrada com a técnica pré-empilhamento em tempo da linha 0204-RL-0058.

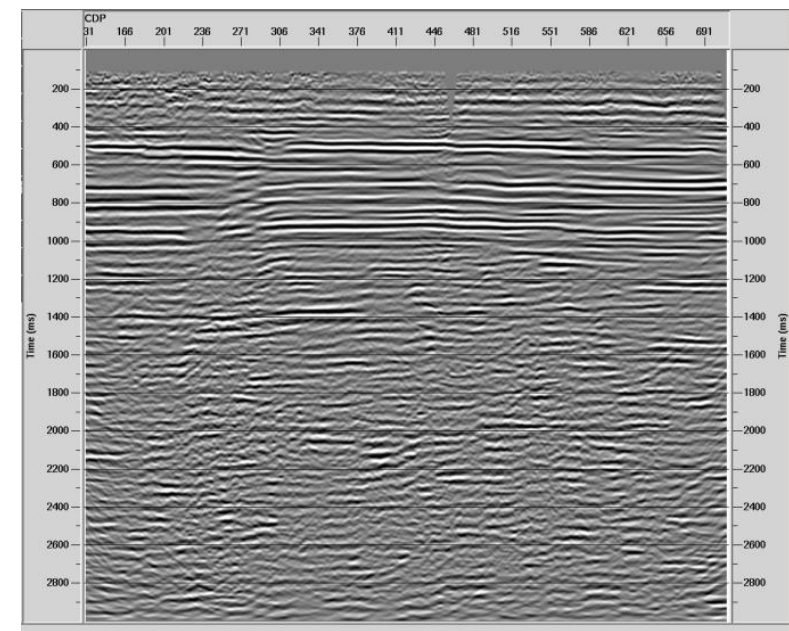

Figura 10 - Seção migrada em tempo após empilhamento CRS da linha 0204-RL-0058.

\section{Discussão e Conclusões}

Apesar da baixa qualidade e da baixa cobertura dos dados sísmicos utilizados no presente trabalho, a fase de pré-processamento (geometria, edição, correção estática, deconvolução e filtragem) foi satisfatória, o que pode ser comprovado pelos bons resultados obtidos tanto com o processamento PreSTM quanto com o processamento não convencional. No entanto, os resultados obtidos com - método de empilhamento CRS se mostraram superiores comparados com os resultados obtidos com a técnica de migração pré-empilhamento em tempo.

O resultado da aplicação do método CRS nos dados da Bacia do Amazonas, em geral, exibe uma razão sinal/ruído superior e melhor continuidade lateral dos refletores, em relação aos resultados obtidos no processamento realizado através do método PreSTM, isto devido ao fato de que o método CRS abrange mais dados para o empilhamento e utiliza um número maior de parâmetros de empilhamento.
Devido ao ganho na qualidade dos resultados finais processados com a técnica de empilhamento CRS apontados nas três linhas sísmicas da Bacia do Amazonas, indica-se o uso desta técnica em posteriores estudos de reprocessamento e interpretação dos dados sísmicos da mencionada bacia.

\section{Agradecimentos}

O primeiro autor agradece ao Convênio UFPA/ANP/MCT/PRH-06, pelo apoio financeiro a qualidade de bolsa de estudo.

À Universidade Federal do Pará, pelos equipamentos e espaços cedidos.

\section{Referências}

BIONDI, B. L. 3-D Seismic imaging. Tulsa: Society of Exploration Geophysics, 2006.

CAPUTO, M. V. Stratigraphy, tectonics, paleoclimatology and paleogeography of Northern Basins of Brazil. 1984. 583f. Tese (Doutorado), University of California, Santa Barbara. 1984.

CLAERBOUT, J. F. Imaging the earth's interior. Oxford: Blackwell Scientific Publications. 1985. 398 p.

EIRAS, J. F.; WANDERLEY FILHO, J.R. Ocorrências de petróleo relacionadas a rochas magmáticas fanerozóicas. In: SIMPÓSIO SOBRE VULCANISMO E AMBIENTES ASSOCIADOS, 2., 2002, Belém. Boletim de resumos e roteiro de excursão. Belém: Núcleo PRONEX do Centro de Geociências da UFPA, 2002. p.54.

GARABITO CALLAPINO, G. Empilhamento sísmico por superfície de reflexão comum: um novo algoritmo usando otimização global e local. 2001. 65f. Tese (Doutorado) Universidade Federal do Pará, Centro de Geociências, Programa de Pós-Graduação em Geofísica, Belém. 2001.

GARABITO CALLAPINO, G.; CRUZ, J. C.; HUBRAL, P.; COSTA, J. Common reflection surface stack: A new parameter search strategy by global optimization. In: SEG MEETING, 71. San Antonio, Expanded Abstracts. p. 2009-2012. 2001.

ISSLER, R. S. et al. Geologia da Folha SA.22 Belém. In: BRASIL. DEPARTAMENTO NACIONAL DE PRODUÇÃO MINERAL. Projeto RADAM. Folha SA.22 Belém. Rio de Janeiro: DNPM, 1974, v.5, p.160.

PASCHOAL JR., G. J. W. Estudo do método de empilhamento SRC e aplicação em dados sintéticos e reais. 2004. 103f. Dissertação (Mestrado)Universidade Federal do Pará, Instituto de Geociências, Programa de Pós-Graduação em Geofísica, Belém, 2005.

TYGEL, M.; MÜLLER, T.; HUBRAL, P.; SCHLEICHER, J. Eigenwave based multiparameter traveltime expansion. In: SEG MEETING, 67, Dallas, Expanded Abstracts, p. 1770-1773. 